Planetary Systems in the Universe - Observation, Formation and Evolution

Proceedings IAU Symposium No. 202, (c)2004 IAU

Alan Penny, Pawel Artymowicz, Anne-Marie Lagrange, \& Sara Russell, eds.

\title{
The Distribution of Asteroidal Dust in the Inner Solar System
}

\author{
Keith Grogan \\ NASA Goddard Space Flight Center, Code 681, Greenbelt, MD 20771, \\ $U S A$
}

\author{
S.F. Dermott and T.J.J. Kehoe \\ University of Florida, Department of Astronomy, Gainesville, \\ FL 32611, USA
}

\begin{abstract}
In this paper we demonstrate how the action of secular resonances near the inner edge of the asteroid belt strongly effects the inclinations and eccentricities of asteroidal dust particles, such that they lose the orbital characteristics of their parent body and are dispersed into the zodiacal background. As a consequence, it may not be possible to relate the distribution of interplanetary material at $1 \mathrm{AU}$ to given asteroidal or cometary sources with the level of confidence previously imagined.
\end{abstract}

\section{Introduction}

The aim of this research is to better understand the dynamical behaviour of main-belt source dust particles over a wide range of particle sizes, and incorporate the results into physically motivated models that describe the brightness distribution of the zodiacal cloud. Previous attempts to model the orbital evolution of these dust particles (Dermott et al. 1996) have been limited to considering particles with diameters less than $25 \mu \mathrm{m}$. This is because larger dust particles take longer (millions of years) to decay into the Sun under the influence of Poynting-Robertson and solar-wind drag than their smaller counterparts (Burns et al. 1979). However, the LDEF (Long Duration Exposure Facility) cratering record (Love and Brownlee 1993) is the latest evidence suggesting a significant population of large interplanetary dust particles (100 $\mu \mathrm{m}$ diameter and greater) near $1 \mathrm{AU}$. It is important to extend our current models of the zodiacal cloud to include this large particle population.

\section{Numerical Simulations}

To achieve this we have developed a unique integration code specifically designed for evolving the orbits of large populations of dust particles under the effects of radiation pressure, Poynting-Robertson drag and solar-wind drag, as well as point-mass gravitational forces. We have applied the dissipative mapping technique described by (Malhotra 1994) to the specific problem of deriving a MVS (Mixed Variable Symplectic) type integration code (Wisdom and Holman 1991) that also incorporates the effects of these non-gravitational forces. The develop- 

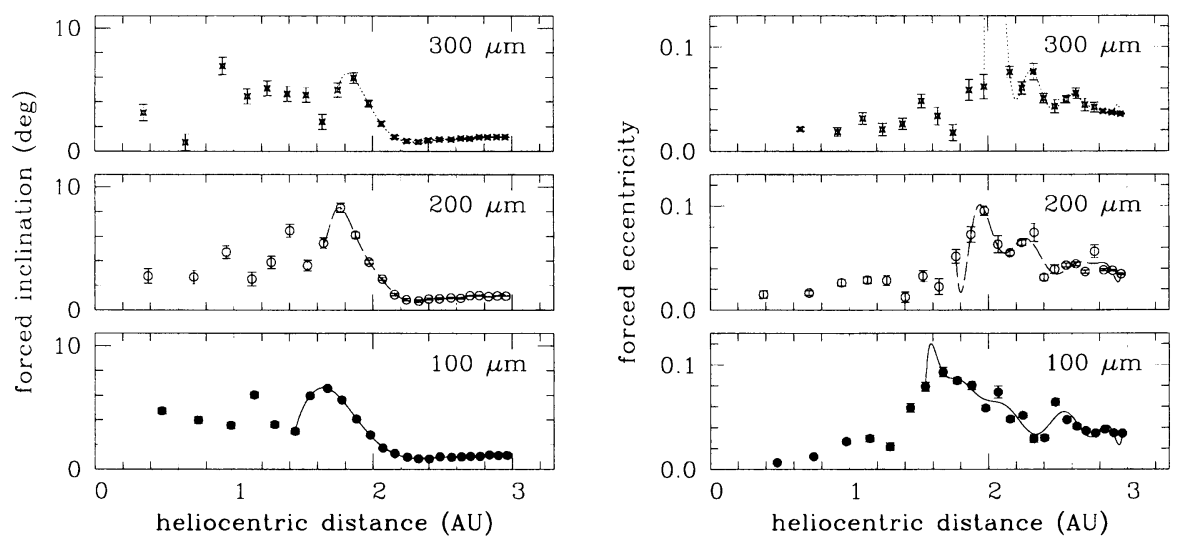

Figure 1. The variation of both the forced inclination and forced eccentricity with heliocentric distance of 100, 200 and $300 \mu \mathrm{m}$ dust particles, originating from the Eos asteroid family.

ment and testing of this dissipative code is described in detail in Kehoe (1999). This code is significantly faster than more conventional integration techniques and so allows the investigation of problems that were hitherto considered to be too computationally expensive.

The new code was employed to calculate the orbital element distribution of large (100 $\mu \mathrm{m}$ and greater) Eos family dust particles at the present epoch. A representative sample of 124 Eos family dust particles was evolved forward in time, along with the Sun and the planets Jupiter, Saturn, Uranus, and Neptune, from 24 different epochs in the past. These 24 past epochs were selected to provide orbital elements for the representative family of Eos dust particles across a wide range of semimajor axis values in the inner Solar System. We considered Eos family dust particles composed of astronomical silicate of density $2,500 \mathrm{~kg} \mathrm{~m}^{-3}$ with diameters 100,200 and $300 \mu \mathrm{m}$, for which we calculated $\beta$ values of $0.00446,0.00221$ and 0.00147 , using Mie theory. Note that our results are valid for any choice of material: the $\beta$ values given here would simply correspond to a different set of particles sizes. The longest integrations performed for the 100,200 and $300 \mu \mathrm{m}$ dust particles were for time scales of $0.6 \mathrm{Myr}, 1.2 \mathrm{Myr}$ and $1.8 \mathrm{Myr}$, respectively.

The results of the integrations presented in the next section represent a total of approximately 2 months of CPU time running on a variety of Pentium processors.

\section{Results}

Figure 1 shows the variation with heliocentric distance of both the forced inclination and fored eccentricity of the 100, 200 and $300 \mu \mathrm{m}$ dust particles. The forced elements are the component of the osculating elements due to perturbations from the planets (Kortenkamp and Dermott 1998). Near the source region, the forced elements of these large particles display similar behaviour to 
that of the smaller dust particles we have previously investigated (Dermott et al. 1996). That is, their forced inclinations and eccentricities are locked onto Jupiter's inclination and eccentricity, respectively. However, as the dust particles encounter the $\nu_{6}$ and $\nu_{16}$ secular resonances at the inner edge of the asteroid belt (at about $2 \mathrm{AU}$ ), the effect of these resonances increases the value of their forced eccentricities and inclinations, respectively. This is particularly significant in the case of the forced eccentricity of the $300 \mu \mathrm{m}$ dust particles, which increases to a value of $\sim 0.8$ just outside $2 \mathrm{AU}$.

\section{Conclusions}

1. The effect of secular resonances becomes important for large dust particles originating in the asteroid belt as their dynamical lifetime increases

2. The secular resonances disperse the orbital elements of the dust particles and in particular their inclinations and eccentricities

3. The orbital element distributions of large asteroidal dust particles produced by asteroidal collisional attrition therefore lose their characteristic family signatures and become indistinguishable from the general background cloud of dust

4. The Solar System dust bands (Grogan et al. 1997) have a natural inner edge at about $2 \mathrm{AU}$ associated with the $\nu_{6}$ and $\nu_{16}$ secular resonances

5. Large asteroidal dust particles in the inner Solar System become indistinguishable from cometary dust particles on the basis of their orbital element distributions

\section{References}

Burns, J. A., Lamy, P. L. \& Soter, S. 1979, Icarus, 40, 1

Dermott, S. F., Jayaraman, S., Xu, Y. L., Grogan, K. \& Gustafson, B. A. S. 1996, in AIP Conference Proc. Vol. 348, Unveiling the Cosmic Infrared Background, ed. E. Dwek (New York: Woodbury), 25

Grogan, K., Dermott, S.F., Jayaraman, S. \& Xu, Y.L. 1997, Planet. Space Sci, 45, 1657

Kehoe, T. J. J. 1999, Long term dynamics of small bodies in the solar system using mapping techniques. Ph.D. thesis, University of London

Kortenkamp, S. J. \& Dermott, S. F. 1998, Icarus, 135, 469

Love, S. G. \& Brownlee, D. E. 1993, Science, 262, 550

Malhotra, R. 1994, Celest. Mech. Dynam. Astron., 60, 373

Wisdom, J. \& Holman, M. 1991, AJ, 102, 1528 